\title{
Calculations of Alfvén Wave Heating in TCABR Tokamak
}

\author{
A.G. Elfimov, R.M.O. Galvão, \\ Institute of Physics, \\ University of São Paulo, 05315-970, SP, Brazil \\ S.A. Galkin, A.A. Ivanov, and S.Yu. Medvedev \\ Keldysh Institute of Applied Mathematics, RAS, Moscow, Russia
}

Received on 3 July, 2001

\begin{abstract}
A two dimensional code ALTOK, which is designed for calculating plasma heating due to radiofrequency fields in the Alfvén and in Ion Cyclotron Ranges of Frequencies in axisymmetric tokamaks, is used to analyze Alfvén wave absorption in multi species plasmas in TCABR (Tokamak Chauffage Alfvén Brasilien) [Nucl.Fusion 30, 503 (1996)]. A good agreement between the results obtained with ALTOK code calculations and with a two dimensional kinetic code [Phys. Plas., 6 (1999) 2437] is shown for Alfvén wave dissipation in hydrogen plasmas. The global Alfvén wave resonance of the $m=0$ mode is found to be the best candidate to explain some heating regimes in TCABR.
\end{abstract}

\section{Introduction}

The method of Alfvén wave (AW) heating is based on the mode conversion effect[1] (named a local AW resonance (LAR) [2, 3]) of the radio frequency (rf) field, induced by an external antenna, into a kinetic AW that effectively dissipates on electrons. The analysis of Alfvén wave absorption in tokamaks with two dimensional $(2 D)$ numerical codes has its origin on the MHD plasma model developed in Ref [4]. Lately, this model was extended to ion cyclotron range of frequencies (ICRF) by including ion cyclotron resonance with collisional dissipation (see Ref [5]). However, the effects of wave dissipation on electrons was modeled by an artificial damping to overcome the logarithmic divergence in LAR. Some kinetic $2 D$-codes for AW heating were developed for the axis-symmetric tokamak plasma geometry $[6,7,8]$. However, nonlocal effects in the kinetic dielectric tensor produced difficulties in the procedure for solving the Maxwell equations numerically, especially in the vicinity of the ion cyclotron resonance.

Recently, the MHD plasma model was extended to a multi-fluid plasma model which includes electron and two species ion fluids [9]. This two-dimensional numerical code (named ALTOK) can help to resolve the problem of numerical solution at the local AW resonance because it includes naturally the electron-ion col- lision dissipation and the electron inertia in the parallel component of the dielectric tensor. In this case, a slow quasi-electrostatic Alfvén wave (SQAW) [10, 11] may appear at the mode conversion magnetic surface. This model is sufficient for analysis of Alfvén and fast magneto-sonic waves in ICRF.

Here, using the ALTOK code, we present relevant results of numerical calculations related to the Alfvén wave heating experiments in TCABR [12], taking into account carbon and oxygen impurities, and compare some results with the corresponding ones obtained through the kinetic code [7].

The paper is organized as follows. In Section II, we briefly describe the toroidal multi-fluid plasma model. In Section III, we present the result of calculations of Alfvén wave dissipation in the TCABR tokamak. Finally, we analyze some heating regimes in TCABR and summarize the main results of our calculations.

\section{Plasma model}

The standard plasma model includes Maxwell equations and the dielectric tensor, which is calculated, in our case, from multi-fluid MHD equations (see, for example [13]). Assuming harmonic dependence in time and in toroidal angle $\varphi$ for the wave magnetic field $\mathbf{B}$, electric field $\mathbf{E}$, and current density $\mathbf{j}$, 


$$
\mathbf{E}=\sum_{n} \mathbf{E}_{n} e^{i n \varphi-i \omega t}, \quad \mathbf{B}=\sum_{n} \mathbf{B}_{n} e^{i n \varphi-i \omega t}, \quad \mathbf{j}=\sum_{n} \mathbf{j}_{n} e^{i n \varphi-i \omega t},
$$

the Maxwell equations can be presented as follows:

$$
\nabla \times \nabla \times \mathbf{E}-\frac{\omega^{2}}{c^{2}}\left(\hat{I}-\frac{4 \pi i}{\omega} \hat{\sigma}\right) \mathbf{E}=\frac{4 \pi i \omega}{c^{2}} \mathbf{j}^{e x t}
$$

where $\mathbf{j}^{\text {ext }}$ is an external driving current in the antenna and generalized Ohm's law $\mathbf{j}=\hat{\sigma} \mathbf{E}$ is used with $\hat{\sigma}$ being the susceptibility tensor in a local bases of coordinates

$$
\begin{gathered}
\mathbf{e}_{\|}=\frac{\mathbf{B}_{0}}{\left|\mathbf{B}_{0}\right|}, \quad \mathbf{e}_{N}=\frac{\nabla \psi}{|\nabla \psi|}, \quad \mathbf{e}_{\perp}=\mathbf{e}_{\|} \times \mathbf{e}_{N}, \\
\frac{4 \pi i}{\omega} \hat{\sigma}=\left(\begin{array}{rrr}
\sigma_{N} & \sigma_{N \perp} & 0 \\
-\sigma_{N \perp} & \sigma_{\perp} & 0 \\
0 & 0 & \sigma_{\|}
\end{array}\right),
\end{gathered}
$$

where $\psi$ is poloidal magnetic flux and tensor components are

$$
\begin{gathered}
\sigma_{N}=\sum_{\alpha} \frac{\omega_{p \alpha}^{2}}{\left(\omega_{B \alpha}^{2}-\omega^{2}\right)}, \quad \sigma_{\perp}=\sum_{\alpha} \frac{\omega_{p \alpha}^{2}}{\left(\omega_{B \alpha}^{2}-\omega^{2}\right)}, \\
\sigma_{N \perp}=\sum_{\alpha} \frac{\omega_{B \alpha} \omega_{p \alpha}^{2}}{\omega\left(\omega_{B \alpha}^{2}-\omega^{2}\right)}, \quad \sigma_{\|}=-\sum_{\alpha} \frac{\omega_{p \alpha}^{2}}{\omega^{2}}-\frac{\omega_{p, e}^{2}}{\omega\left(\omega+\mathrm{i} \nu_{e}\right)}
\end{gathered}
$$

In the equations $\nu_{e}$ is the electron-ion collision frequency, $\omega_{p \alpha}$, and $\omega_{B \alpha}$ are the plasma and cyclotron frequencies, respectively, for species $\alpha$. The wave absorption power density is given by $W=\operatorname{Re}\left(\mathbf{j} \cdot \mathbf{E}^{*}\right)$.

A general two dimensional grid with non-orthogonal quadrangular cells is used to discretize Eq.(2). For the axis-symmetric equilibrium, a quasi radial-annulus grid, adapted to magnetic surfaces, is applied. The equilibrium code POLAR-2D [14] is employed to solve the Grad-Shafranov equation for $\psi$ and obtain the grid adapted to magnetic surfaces. However an arbitrary grid can be also used if the equilibrium magnetic field is directly specified.

\section{Alfvén Wave Heating}

Here, using the $2 D$ kinetic [7] and MHD codes[9], we present relevant results of numerical calculations on the AW heating experiments in TCABR (see the discharge 4893 in Ref [12]) and compare them with old TCA results [15]. The simulations have been carried out assuming a circular cross-section tokamak geometry with the following parameters: minor radius $a=0.18 \mathrm{~m}$; major radius $R_{0}=0.615 \mathrm{~m}$; antenna surface radius $b=0.2 \mathrm{~m}$; wall radius $d=0.23 \mathrm{~m}$, toroidal magnetic field $B_{0}=1.1 T$, plasma current $I_{p}=54 k A$, for the ohmic stage, and $I_{p}=57 k A$, for the rf stage. The kinetic code calculations are carried out assuming a temperature profile given by $T_{\alpha}=T_{\alpha 0}\left(1-r^{2} / a^{2}\right)^{2}$, with $\alpha=e, i, T_{e 0}=500 \mathrm{eV}$ and $T_{i 0}=160 \mathrm{eV}$, respectively. We chose the values of the safety factor to be in the interval $1.1 \leq q_{0} \leq 1.6$ at the magnetic axis, and $4.4 \leq q_{0} \leq 6.4$, at the plasma boundary. These parameters are confirmed with ASTRA transport code[16] calculations (see discussion below). Finally, the electron density profile is assumed to be of parabolic form with a maximum central density $n_{0}=3.2 \times 10^{19} \mathrm{~m}^{-3}$ and a pedestal value $n_{a}=1 \times 10^{18} \mathrm{~m}^{-3}$. The impurity and ion profiles are taken the same. We assume a helical an- 
tenna with poloidal $M=-1$ and toroidal $N=-2 \ldots-6$ wave numbers. The real structure of new TCABR antenna system is taken into account through coefficients calculated in Ref.[17]. In our calculations, we consider only one antenna mode that corresponds to the main component given by the Fourier analysis of the actual antenna system.

Because of small pressure corrections and to simplify calculations, we use a force-free equilibrium in the ALTOK code calculations with the toroidal current density $r j_{\varphi}=\left(1-\bar{\psi}^{\alpha_{2}}\right)^{\alpha_{1}}$ and the plasma density is $n=n_{0}(1-\bar{\psi})^{\alpha_{3}}$, where $\bar{\psi}$ is normalized poloidal magnetic flux function, so that $\bar{\psi}=0$ at the axis and $\bar{\psi}=1$ at the plasma boundary. To have the plasma density and current profiles adjusted to the profiles used in the kinetic code, we use $\alpha_{1}=1.6, \alpha_{2}=0.95$ and $\alpha_{3}=0.7$. In Fig.1, we show that there is reasonable coincidence between the corresponding plasma profiles of the ALTOK and kinetic code for the poloidal angle $\pi / 2$.
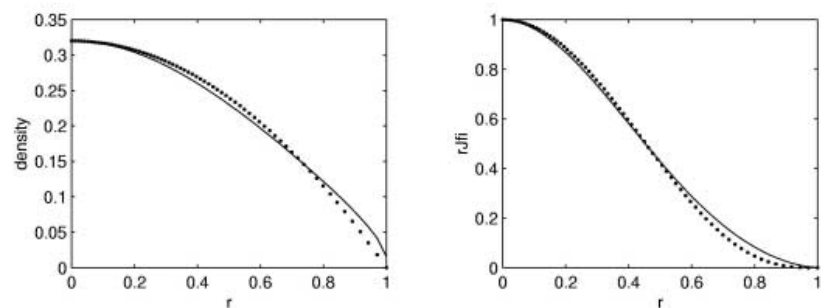

Figure 1. Density $n_{e}$ and toroidal current profile $r j_{\varphi}$ over the radius at the poloidal angle $\pi / 2$ of tokamak crossection; the solid lines are the ALTOK code profiles, the dotted lines mark profiles used in the kinetic code.

Here we consider wave dissipation in the Alfvén wave continuum. Generally, in the cylindrical model, the equation that governs the Alfvén wave continuum can be written in the form,

$$
\omega^{2} \epsilon_{\perp}(r)=k_{\|}^{2}(r) c^{2}, \epsilon_{\perp}=\sum_{\alpha} \frac{\omega_{p \alpha}^{2}}{\left(\omega_{c \alpha}^{2}-\omega^{2}\right)}
$$

For hydrogen plasmas, the equation for the Alfvén wave continuum can be simplified to

$\omega_{A}=\frac{k_{\|} c_{A}}{\sqrt{1+k_{\|}^{2} c_{A}^{2} / \omega_{c, i}^{2}}}, \quad c_{A}=\frac{B_{0}}{\sqrt{4 \pi n_{e} m_{i}}}, \quad k_{\|}=\frac{(n q+m)}{R_{0} q}$

In Fig.2, we show the $M=-1, N=-4$-antenna impedance for pure hydrogen plasmas and plasmas with carbon impurity (capital letters are used for antenna vacuum modes and lower case are used for modes actually excited in the plasma). We can observe spikes of the impedance related to the $m=0$ and $m=-1$ global wave resonances situated below Alfvén wave continuum. The corresponding wave dissipated power, calculated with the ALTOK code for frequencies $f=$ $4.2 \mathrm{MHz}$ and $4.6 \mathrm{MHz}$, is presented in Fig.3. Usually, the $q$ profile is difficult to control in TCABR experiments but the $m=0$-local Alfvén resonance (8) for hydrogen plasmas does not depend on the $q$ profile. In this case, the dissipated wave power presented in Fig.3a is strongly peaked in the plasma core and it can be effectively used for plasma core heating. Note that our calculations confirm the global Alfvén wave resonance that was fond experimentally in old TCA deuterium plasmas [15] with the parameters: $B_{0}=1.2 \mathrm{~T}, n_{0}=2.6 \times 10^{19} \mathrm{~m}^{-3}, q_{0}=1.1$, $M=-1, N=-2, f=2.5 \mathrm{MHz}$.

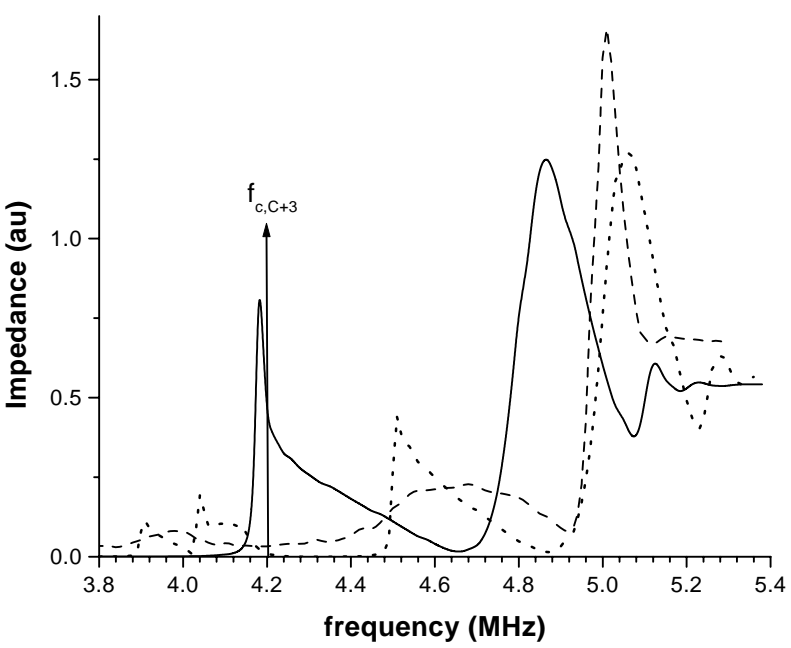

Figure 2. The antenna loading impedance $I_{M N}$ plotted as a function of the generator frequency $f$ for poloidal and toroidal wave numbers $M=-1, N=-4$ of the antenna spectrum. The impedance curves are calculated with the kinetic code (solid for pure hydrogen, doted with 0.2impurity plasmas) and dashed line correspond the MHD code calculations for plasma with $0.2 \%$-carbon impurity The plasma parameters are $n_{0}=3.2 \times 10^{19} \mathrm{~m}^{-3}, T_{e 0}=500 \mathrm{eV}, B_{t}=1.1 \mathrm{~T}$, $q_{0}=1.1, q_{a}=4.4$.

Because of an uncontrollable increase of the light impurities, such as carbon and oxygen, supplied by the chamber wall during the rf pulse, the global wave frequency and Alfvén wave continuum can be strongly modified if the Alfvén wave frequency is about of the ion cyclotron frequency. In Fig.4, we show the cylindrical Alfvén continuum (7) modification in a plasma with light impurities in shot \# 4893. This situation is also calculated using $2-D$ codes. For example, in TCABR hydrogen plasma with $0.2 \%$ of three times ionized carbon (or four times ionized oxygen), the cyclotron frequency is $f=4.2 \mathrm{MHz}$ and it can affect Alfvén wave dissipation. That effect is also demonstrated in Fig. 2 where the Alfvén wave continuum is destroyed for frequencies about this cyclotron frequency. In this case, the better heating regimes can be occur about $f=4.6 \mathrm{MHz}$ that is above the $C_{12}^{+3}$-cyclotron frequency (see Fig.3b). 

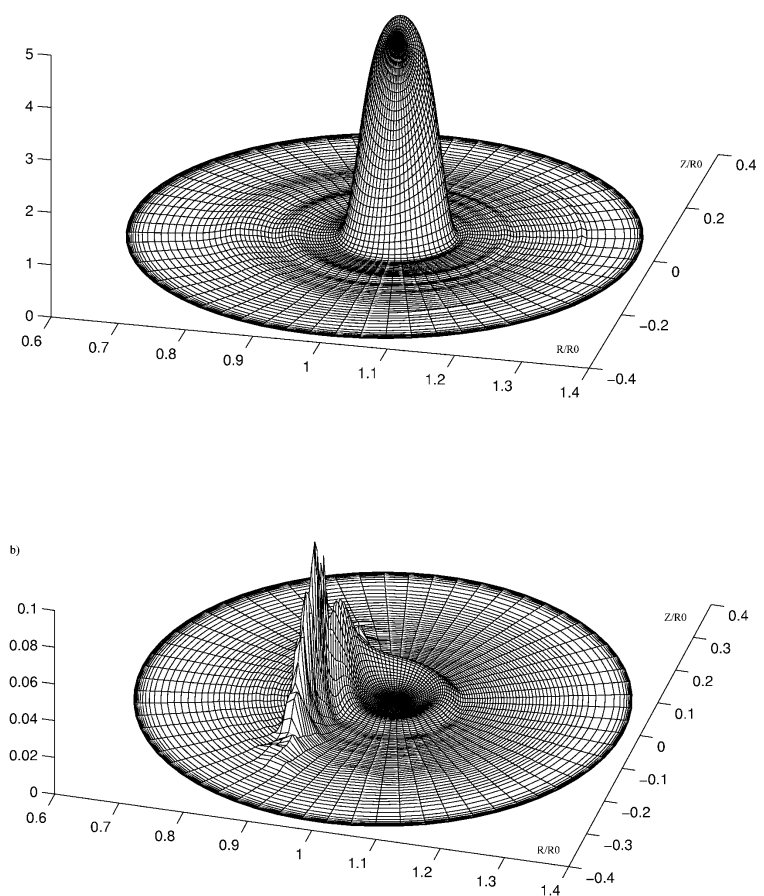

Figure 3ab. Distribution of dissipated wave power over tokamak cross-section in the spike of the $m=0$-GAW resonance in hydrogen plasmas shown for $f=4.2 \mathrm{MHz}$ (a) and $f=4.6 \mathrm{MHz}$ with 0.2 calculations with $M=-1, N=-4$ antenna configuration for central plasma density $n_{0}=3.2 \times$ $10^{19} \mathrm{~m}^{-3}$.

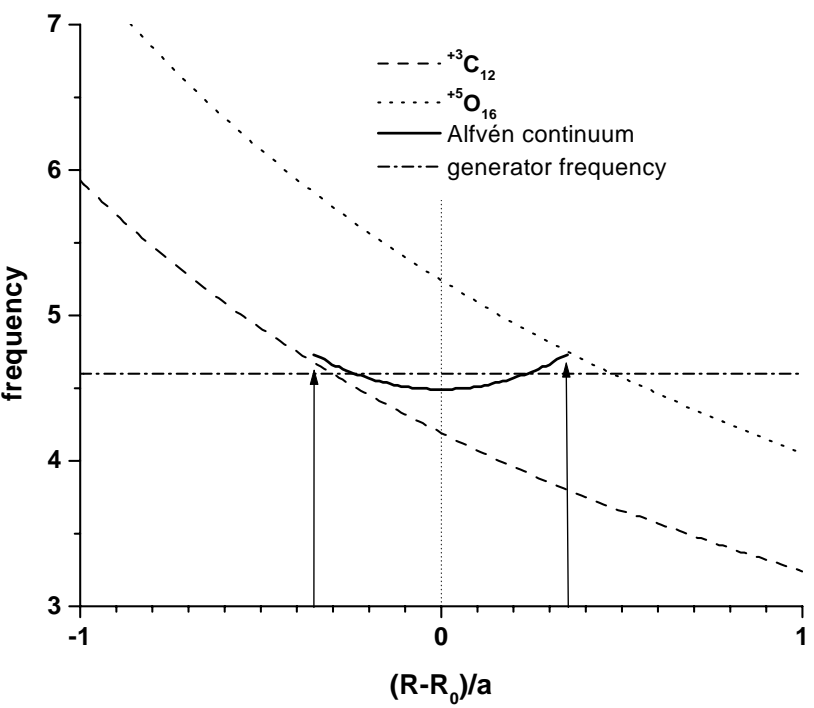

Figure 4. Plot of the cylindrical Alfvén continuum taken from Eq (7) and different carbon and oxygen impurity cyclotron resonances in hydrogen plasmas for conditions in Fig.2.
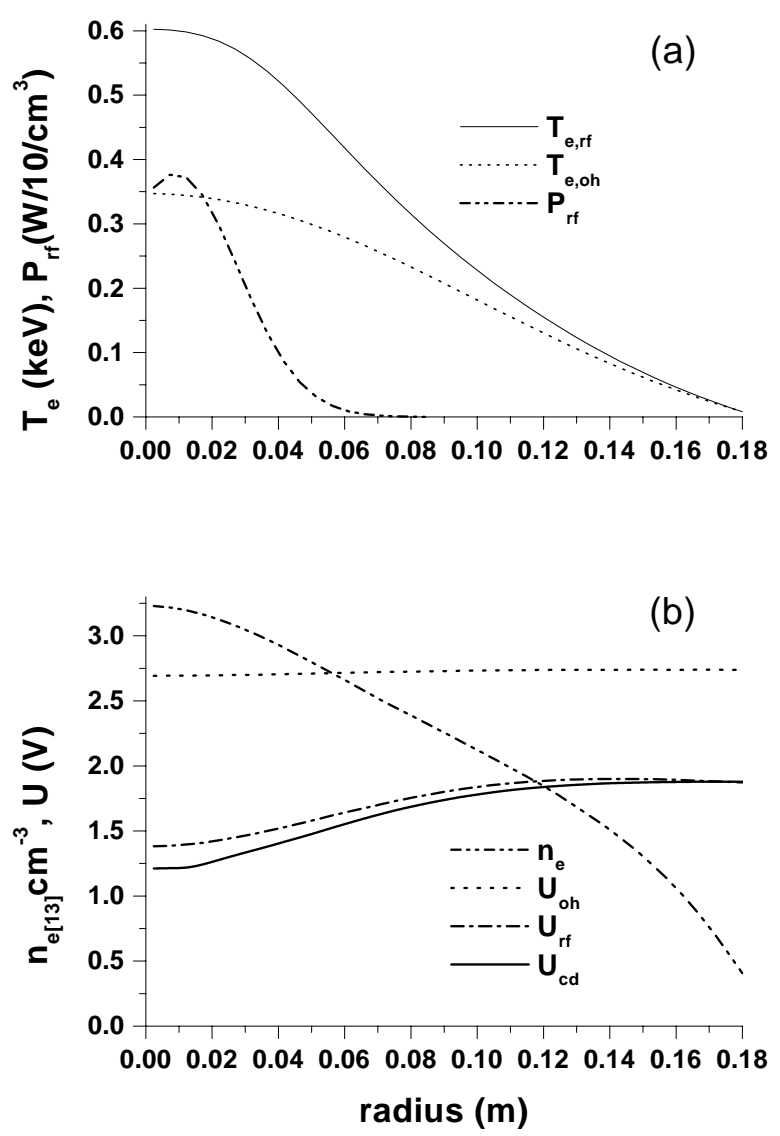

Figure 5. Distribution of electron temperature (a), loop voltage and dissipated wave power is calculated with the ASTRA code and shown for ohmic $\left(t_{1}=53 \mathrm{~ms}\right.$, dotted lines $)$ and $\mathrm{rf}\left(t_{2}=61.5 \mathrm{~ms}\right.$, dash lines) stage in $\mathrm{N} 4893$ discharge. Loop voltage under $\mathrm{rf}$ power is shown with $\mathrm{rf}$ heating (dashdot line) and taking into account $1.2 \mathrm{kA}$ current drive (solid line). 


\section{Conclusion}

In conclusion, we can say:

- Alfvén wave absorption of coupled side-bands harmonics exited by $M=-1$ antenna causes a broad power deposition at $\mathrm{m}=0$ local $\mathrm{AW}$ resonance that surpass the power deposition of global AW at the plasma center;

- choosing generator frequency properly in $m=0$ Alfvén wave continuum, Alfvén wave absorption can be concentrated in the plasma core;

- the ASTRA code calculations confirm Alfvén wave heating $\Delta T_{e}(0) \approx 250 \mathrm{eV}$ and non-inductive current $\Delta I_{c d} \approx 1.2 \mathrm{kA}$ driven by one module antenna with $60 \mathrm{~kW}$ dissipated power in the plasma.

- to analyze the AW dissipation properly, the effect of ion cyclotron resonance zones introduced by partially ionized impurities should be taken into account.

Acknowledgments Authors thankful to Dr.G.V.Pereverzev for the help with ASTRA calculations. This work is supported by the Ministry of Science and Technology/Brazil, through Pronex Project and FAPESP (Foundation of the State of São Paulo for the Support of Research).

\section{References}

[1] V.V. Dolgopolov, K.N. Stepanov, Nuclear Fusion 5, 276 (1965).

[2] J. Tataronis and W. Grossman, Z. Phys. 261, 203 (1973).

[3] A. Hasegawa and Ch. Uberoi, The Alfvén wave (Technical Information Center U.S. DOE, 1982).

[4] K. Appert, B. Balet, R. Gruber et al., Nucl. Fusion 22, 903 (1982).

[5] L. Villard, K. Appert, R. Gruber and J. Vaclavik Comp. Phys. Rep. 4, 95 (1986).
[6] M.V. Dmitrieva, A.A. Ivanov, A.V. Sidorova, et al. in: 15th European Confer. Controlled fusion and plasma physics. Dubrovnik, May (1988), part III, p.944.

[7] G. Amarante-Segundo, A.G. Elfimov, D.W. Ross, R.M.O. Galvão, I.C. Nascimento Phys. Plasmas, 6, 2437 (1999).

[8] A. Jaun in Recent Research Developments in Plasmas (Transworld Research Publishing, Trivandrum, Kerala, India, 2000).

[9] S.A. Galkin, A.A. Ivanov, S.Yu. Medvedev and A.G. Elfimov, Multi Fluid MHD Model And Calculations Of Alfvén Wave Spectrum And Dissipation In Tokamaks. To be published in Comp. Phys. Communications (2002).

[10] D.W. Ross, G.L. Chen and S.M. Mahajan Phys. Fluids 25, 652 (1982).

[11] J. Vaclavik and K. Appert Nucl Fusion 31, 1945 (1991).

[12] L.F. Ruchko, E. Lerche, R.M.O. Galvão et al. The Analyses of Alfvén Current Drive and Wave Heating in TCABR Tokamak. To be published in this issue Brazilian J. of Physics (2002).

[13] V.L. Ginsburg Propagation of Electromagnetic Waves in Plasma (Gordon and Breach, New York, 1961)

[14] L. Degtyarev, A. Martynov, S. Medvedev et al. Comput. Phys. Commun. 103, 10 (1997).

[15] G.A. Collins, F. Hofmann, B. Joye et al., Phys. Fluids, 29, 2260 (1986).

[16] G.V. Pereverzev, P.N. Yushmanov, A.Yu. Dnestrovskii et al. ASTRA, An Automatic System for Transport Analysis in a Tokamak, IPP 5/42, Max-Planck Institute fur Plasmaphysik EUROATOM Association, D8046 Garching, Germany, Aug.1991.

[17] L.F. Ruchko, E. Ozono, R.M.O. Galvão, I.C. Nascimento, F.T. Degasperi, E. Lerche, Fusion Eng. Des. 43, 15 (1998). 